\title{
Rastreio do aneurisma da aorta abdominal - revisão baseada na evidência
}

Vítor Portela Cardoso,* Paula Mendes**

\section{RESUMO}

Objetivo: Rever a evidência sobre a mortalidade e os efeitos laterais do rastreio do aneurisma da aorta abdominal (AAA) em adultos assintomáticos.

Fontes de Dados: National Guideline Clearinghouse, Guidelines Finder, Canadian Medical Association Practice Guidelines, Cochrane, DARE, Bandolier, MEDLINE e Índex de Revistas Médicas Portuguesas.

Métodos: Realizou-se uma pesquisa de meta-análises (MA), revisões sistemáticas (RS), ensaios clínicos aleatorizados e controlados (ECAC) e normas de orientação clínica (NOC), publicados entre 01/01/2003 e 23/03/2013, nas línguas portuguesa, espanhola, inglesa e francesa, utilizando os termos $\mathrm{MeSH}$ : abdominal aortic aneurysm e mass screening. Adotou-se a escala Oxford Centre for Evidence-Based Medicine (CEBM) para avaliação da qualidade dos estudos e atribuição dos níveis de evidência (NE). Resultados: Foram obtidos 337 artigos, tendo sido incluídas 2 MA, 1 RS e 7 NOC. A RS e as MA selecionadas demonstraram que o rastreio diminuía significativamente a mortalidade por AAA nos homens com idades entre os 65-75 anos, mas não nas mulheres. A diminuição da mortalidade por todas as causas foi observada numa MA, tendo sido estimado um número necessário para rastrear de 156, inferior a programas de rastreios oncológicos atualmente instituídos. O rastreio duplicou o número de cirurgias eletivas e diminuiu para cerca de metade o número de cirurgias de emergência por rotura de AAA.

Conclusões: A evidência encontrada foi consistente e de boa qualidade, demonstrando a redução da mortalidade por AAA através do rastreio do AAA em homens assintomáticos com idades $\geq 65$ anos (NE1). O tratamento do AAA está associado a riscos significativos de morte operatória e complicações que poderão ser aceitáveis nos indivíduos de alto risco.

Palavras-chave: Aneurisma da Aorta Abdominal; Rastreio; Mortalidade; Efeitos Laterais.

\section{INTRODUÇÃO}

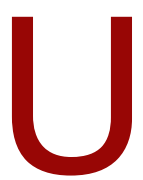

m aneurisma arterial é definido como uma
dilatação localizada maior ou igual a $50 \%$ do
diâmetro normal. ${ }^{1}$ Como o diâmetro da ar-
téria aorta infrarrenal é usualmente de $2 \mathrm{~cm}$, considera-se um AAA quando o diâmetro da aorta abdominal infrarrenal é maior ou igual a $3 \mathrm{~cm} .^{2-5}$

Os valores da prevalência de AAA foram resultado de estudos de rastreio em vários países e variam entre 4$-9 \%$ nos homens e $1 \%$ nas mulheres, aumentando com a idade. ${ }^{3,6-7}$ Estima-se que, de todas as mortes em ho-

*Interno de Medicina Geral e Familiar. Mestrado Integrado em Medicina pela Escola de Ciências da Saúde da Universidade do Minho. Unidade de Saúde Familiar Gualtar. **Interna de Medicina Geral e Familiar. Mestrado Integrado em Medicina pela Escola de Ciências da Saúde da Universidade do Minho. Unidade de Saúde Familiar Maxisaúde. mens com mais de 65 anos, $1-2 \%$ sejam causadas pela rotura de um AAA até então desconhecido. ${ }^{6}$

São fatores de risco major para o AAA a idade avançada, o sexo masculino, o tabagismo e a história familiar. ${ }^{1,4} \mathrm{O}$ risco de AAA é 2-4 vezes superior nos familiares de primeiro grau de parentesco do sexo masculino a pacientes com AAA. ${ }^{1}$ Nos familiares do sexo feminino os dados são contraditórios. ${ }^{1,3}$ Outros fatores de risco associados ao desenvolvimento de AAA parecem ser: a história de outros aneurismas vasculares, estaturas mais elevadas, doença arterial coronária, doença cerebrovascular, aterosclerose, hipercolesterolemia e hipertensão arterial - apesar de a informação dos estudos para alguns destes fatores ser inconsistente. ${ }^{4}$ A diabetes mellitus, os indivíduos do sexo feminino ou de raça negra ou asiática estão associados a um menor risco de desenvolvimento de AAA., ${ }^{4,7}$ 
O AAA forma-se através de mecanismos biológicos complexos ainda não totalmente compreendidos, manifestados pela degeneração da parede do vaso. ${ }^{3} \mathrm{~A}$ história natural dos aneurismas arteriais caracteriza-se pela expansão gradual e/ou esporádica do seu diâmetro e pela acumulação de trombos murais causados pelo fluxo sanguíneo turbulento na periferia. Estas características contribuem para as três complicações mais comuns dos aneurismas: rotura, eventos isquémicos trombo-embólicos e a compressão ou a erosão de estruturas adjacentes, as quais são habitualmente bastante específicas na sua localização. ${ }^{3}$

Mais de $80 \%$ dos AAA são assintomáticos e frequentemente detetados como um achado ecográfico incidental. ${ }^{5}$ Quando sintomáticos, a dor é a queixa mais frequente e geralmente situa-se no hipogastro ou na região dorsal/lombar. ${ }^{3}$ É usualmente constante, com duração de horas a dias e caracterizada como "a roer". ${ }^{3}$ A rotura caracteriza-se por um início abrupto de uma dor nas costas e abdominal, sendo que a tríade patognomónica de dor abdominal/dorsal, massa abdominal pulsátil e hipotensão apenas ocorre em cerca de um terço dos casos. $^{3}$ A sintomatologia de uma rotura de aneurisma pode simular uma cólica renal, diverticulite ou uma hemorragia gastrointestinal, levando assim a erros de diagnóstico que podem custar um tempo precioso. ${ }^{3} \mathrm{Um}$ exame físico completo deve incluir a palpação do abdómen e das artérias dos membros inferiores na tentativa de detetar pulsos alargados que sugerem a presença de aneurismas. ${ }^{3}$ A palpação dos AAA é segura e não foi relatada como precipitante de rotura. ${ }^{3}$ Porém, a deteção de uma massa abdominal pulsátil não é específica da presença de um AAA, podendo esse achado corresponder a uma aorta abdominal tortuosa ou à transmissão das pulsações da aorta a uma massa não-vascular. ${ }^{1}$

Apesar do comportamento habitualmente silencioso do AAA, se não tratado estima-se que um em cada três AAA sofra uma rotura ${ }^{2}$ - evento ao qual está associado uma mortalidade de 50 a $80 \% .{ }^{6}$ Nos homens, quase todas as mortes por rotura de AAA ocorrem acima dos 65 anos de idade e quase todas as mortes relacionadas com o AAA ocorrem abaixo dos 80 anos. ${ }^{7}$ Nas mulheres, a maioria das mortes relacionadas com o AAA ocorre após os 80 anos.? A probabilidade de rotura de um AAA depende do seu tamanho, sendo este o fator de risco independente mais importante. ${ }^{3-4,7-9}$ Outros são: sexo feminino, tabagismo, hi- pertensão arterial, taxa de expansão do AAA e o pico de stress da parede do AAA. ${ }^{4}$ Nos 5 anos que se seguem ao diagnóstico, a rotura ocorre em aproximadamente $2 \%$ dos aneurismas com menos de $4 \mathrm{~cm}$ e em cerca de $25 \%$ naqueles maiores que $5 \mathrm{~cm}$. Assim, após o diagnóstico de AAA, duas abordagens são prática corrente: cirurgia eletiva nos aneurismas de grandes dimensões $(\geq 5,5 \mathrm{~cm})$ ou vigilância ecográfica regular naqueles $c o m<5,5 \mathrm{~cm}$, orientando para a cirurgia caso o aneurisma cresça mais do que $1 \mathrm{~cm} /$ ano ou atinga os $5,5 \mathrm{~cm} .^{3-4,8-9}$

$O$ benefício de se identificarem os AAA precocemente assenta na possibilidade de poderem ser reparados cirurgicamente antes de ocorrer uma potencial complicação, diminuindo assim a mortalidade por AAA. ${ }^{6,9}$ A taxa de mortalidade após rotura de um AAA atinge os $80 \%$, o que é muito superior à taxa de mortalidade hospitalar da cirurgia eletiva de reparação do AAA, que ronda os $4,2 \% .{ }^{10} \mathrm{~A}$ esta cirurgia está ainda associada uma taxa de complicações de $32,5 \%$, algumas importantes como o enfarte agudo do miocárdio. ${ }^{2} \mathrm{Obe}-$ nefício do rastreio será, assim, máximo nos indivíduos com alto risco de desenvolver um AAA suficientemente grande para ter indicação cirúrgica. ${ }^{7}$

A ecografia abdominal é o exame recomendado para o rastreio do AAA, uma vez que a sensibilidade é de $95 \%$ e a especificidade aproxima-se dos $100 \%$, é de fácil acesso, não invasivo, com um preço relativamente baixo e inócuo de radiações ou outros riscos. ${ }^{1-2,4,11}$ Apresenta, ainda assim, desvantagens como o facto de ser um exame operador dependente e poder ser limitado pelo biótipo do doente. ${ }^{4}$ Não existem contraindicações absolutas para a realização de ecografia abdominal; porém, na suspeita de dissecção ou rotura aneurismática a ecografia não é o exame de eleição. ${ }^{8}$

Atualmente são várias as organizações científicas internacionais que recomendam o rastreio do AAA através da ecografia ${ }^{6,12-13} \mathrm{e}$, em 2008, foi iniciado no Reino Unido um programa de rastreio nacional com base nos procedimentos do ensaio clinico MASS. ${ }^{14}$ Paralelamente foram iniciados programas similares na Suécia e nos Estados Unidos da América (Medicare). ${ }^{7,15}$ Em Portugal, entre junho de 2011 e março de 2012, foi levado a cabo o primeiro rastreio nacional do AAA, promovido pela Sociedade Portuguesa de Angiologia e Cirurgia Vascular, destinado a homens com mais de 65 anos de idade e com fatores de risco de AAA. ${ }^{16}$ 
Atendendo a estes factos, a questão que se coloca neste trabalho é se em adultos assintomáticos o rastreio do AAA reduz a mortalidade por AAA e por todas as causas, sem efeitos laterais valorizáveis.

\section{MÉTODOS}

Foi realizada uma pesquisa de meta-análises (MA), revisões sistemáticas (RS), ensaios clínicos aleatorizados e controlados (ECAC) e normas de orientação clínica (NOC) nas fontes de dados: National Guideline Clearinghouse, Guidelines Finder, Canadian Medical Association Practice Guidelines, Direção-Geral da Saúde, Cochrane, DARE, Bandolier, MEDLINE e Índex de Revistas Médicas Portuguesas, publicados entre 1 de janeiro de 2003 e 23 de março de 2013, nas línguas portuguesa, inglesa, francesa e espanhola, utilizando os termos MeSH: abdominal aortic aneurysm e mass screening; ou os termos DeCS: aneurisma da aorta abdominal e rastreio.

Os critérios de inclusão dos artigos consistiram numa população adulta, assintomática, cuja intervenção fosse o rastreio do AAA, comparativamente a não rastrear. Os resultados avaliados foram a mortalidade por todas as causas e a mortalidade relacionada com o AAA. Os resultados secundários foram os efeitos laterais do rastreio. Não foram avaliados os resultados orientados para a qualidade de vida ou a relação custo-efetividade. Foram excluídos os estudos que tivessem tido como alvo crianças, indivíduos sujeitos anteriormente a reparação de um AAA ou com doenças graves/terminais.

Foi adotada a escala "Oxford Centre for Evidence-Based Medicine (OCEBM) 2011" para avaliação da qualidade dos estudos e atribuição dos níveis de evidência (NE). ${ }^{17}$

\section{RESULTADOS}

Obtiveram-se 337 artigos tendo sido selecionadas 1 RS, ${ }^{9} 2$ MA $^{18-19}$ e 7 NOC, ${ }^{3-5,7,22-24}$ após exclusão dos artigos que não cumpriam os critérios de inclusão, os artigos repetidos e os estudos originais que foram alvo de análise nas RS ou MA incluídas na presente revisão.

As RS e MA selecionadas incluíam os mesmos 4 ECAC. Tratou-se de ECAC não cegos que avaliaram o rastreio do AAA de base populacional. Dois tiveram lugar no Reino Unido (Chichester e MASS), um na Dinamarca (Viborg) e um na Austrália (Western Australia). Os participantes foram distribuídos aleatoriamente por computador para receber o convite do rastreio, ou não, e as informações sobre a mortalidade foram colhidas a partir da combinação de dados hospitalares e registos nacionais. O seguimento variou entre 3,5-15 anos, não tendo sido reportada uma perda significativa no seguimento em nenhum ECAC. Apesar destes ensaios terem sido realizados em diferentes países e com diferentes coortes de pacientes, os resultados não tiveram grande divergência. ${ }^{18}$ A United States Preventive Services Task Force (USPSTF) classificou como Good o estudo MASS e como Fair os restantes 3 ECAC. ${ }^{7}$

A RS selecionada tem a chancela da Cochrane (quadro I) e demonstrou que o rastreio diminuía significativamente a mortalidade por AAA nos homens, mas não nas mulheres. ${ }^{9}$ Cosford et al demonstraram que a redução da mortalidade por AAA se devia essencialmente à diminuição na incidência de roturas aneurismáticas verificada nos homens $(O R=0,45$; IC95\% 0,21-0,99), o que não aconteceu nas mulheres. ${ }^{9}$ Não foi detetado um efeito significativo na redução da mortalidade para todas as causas. ${ }^{9}$ Uma RS da USPSTF (Fleming et al), publicada anteriormente, tinha já obtido resultados sobreponíveis. ${ }^{2}$ O tabagismo associou-se a um aumento de 3-5 vezes na prevalência de AAA e no aumento do risco da mortalidade relacionada com AAA. ${ }^{2}$ Estimaram ainda que, se se rastreassem apenas esses homens, a redução da mortalidade para o AAA seria $89 \%$ daquela obtida no rastreio de todos os homens com idades entre os 65 e 74 anos. $^{2}$

A RS da Cochrane não refere malefícios físicos associados ao rastreio através de ecografia. ${ }^{9}$ Porém, ocorreu um aumento significativo das cirurgias eletivas a AAA nos homens (OR=2,03, IC95\% 1,59-2,59), procedimento que se associou a uma morbi-mortalidade significativa, com uma taxa de mortalidade de $4,2 \%$ e uma taxa de complicações de $32,4 \%$ (enfarte agudo do miocárdio, insuficiência respiratória e renal e alterações do estado funcional dos indivíduos). ${ }^{2,9} \mathrm{O}$ risco de morte e complicações neste contexto aumentava com a idade..$^{2,9}$

Takagi et al publicaram, em 2010, uma MA (quadro II) que incluiu apenas os dados do seguimento a longo prazo (acima dos 10 anos) dos 4 ECAC descritos. ${ }^{18} \mathrm{~A}$ fim de maximizar a homogeneidade do estudo, a MA deste grupo incluiu unicamente os indivíduos do sexo masculino, excluindo o único ECAC que analisou mulheres (Chichester). Os autores demonstraram o efeito na redução da mortalidade relacionada com AAA de $4 \mathrm{em}$ 


\section{QUADRO I. Revisão Sistemática}

\begin{tabular}{|c|c|c|c|c|}
\hline Referência & População & Intervenção & Resultados & $\begin{array}{l}\text { Nível de } \\
\text { evidência }\end{array}$ \\
\hline $\begin{array}{l}\text { Cosford et al, } \\
2007^{9} \\
\text { Reino Unido } \\
4 \text { ECAC }\end{array}$ & $\begin{array}{l}n=137233 \\
(127891 \\
\text { homens) } \\
65-83 \text { anos } \\
\text { Seguimento } \\
\text { de } 2,5-5,1 \\
\text { anos }\end{array}$ & $\begin{array}{l}\text { Rastreio do AAA } \\
\text { em adultos } \\
\text { assintomáticos } \\
\text { por ecografia } \\
\text { abdominal }\end{array}$ & $\begin{array}{l}\text { Mortalidade } \\
\text { Diminuição significativa da } \\
\text { mortalidade por AAA nos homens } \\
\text { com idades entre os } 65 \text { e } 79 \text { anos } \\
\text { (OR=0,60, IC95\% } 0,47-0,78 \text { ), mas } \\
\text { não nas mulheres } \\
\text { Sem diferenças significativas na } \\
\text { mortalidade para todas as causas } \\
\text { (OR=0,95, IC95\% } 0,85-1,07 \text { ) } \\
\text { Efeitos laterais } \\
\text { Aumento significativo das } \\
\text { cirurgias a AAA nos homens } \\
\text { (OR=2,03, IC95\% } 1,59-2,59 \text { ) }\end{array}$ & 1 \\
\hline
\end{tabular}

Legenda: AAA - Aneurisma da Aorta Abdominal; ECAC - Ensaio Clínico Aleatorizado e Controlado; IC - Intervalo de Confiança; OR - Odds Ratio.

1.000 homens com idades $\geq 65$ anos, atribuindo-lhe um número necessário para rastrear (NNR) de $238 .{ }^{18}$ No que diz respeito ao efeito do rastreio em diminuir a mortalidade por todas as causas afirmavam que, ape- sar de verificarem uma forte tendência, o rastreio a longo prazo não diminuía significativamente esta mortalidade $(\mathrm{NNR}=217) \cdot{ }^{18}$ Após a publicação do artigo final do ECAC MASS, em outubro de 2012, ${ }^{14}$ Takagi et al atualizaram a sua MA (quadro II), que apenas analisou a mortalidade por todas as causas. Concluíram que o rastreio diminuía significativamente essa mortalidade em 2,7\% (OR=0,973; IC95\% 0,950,997 ) em homens com idades $\geq 65$ anos, atribuindo-lhe um NNR de $156 .{ }^{19}$ Esta conclusão já tinha sido obtida numa MA anterior (Lindholt et al); ${ }^{10}$ porém, os seus resultados foram muito contestados, tendo vários investigadores apontado erros não só a esta MA,

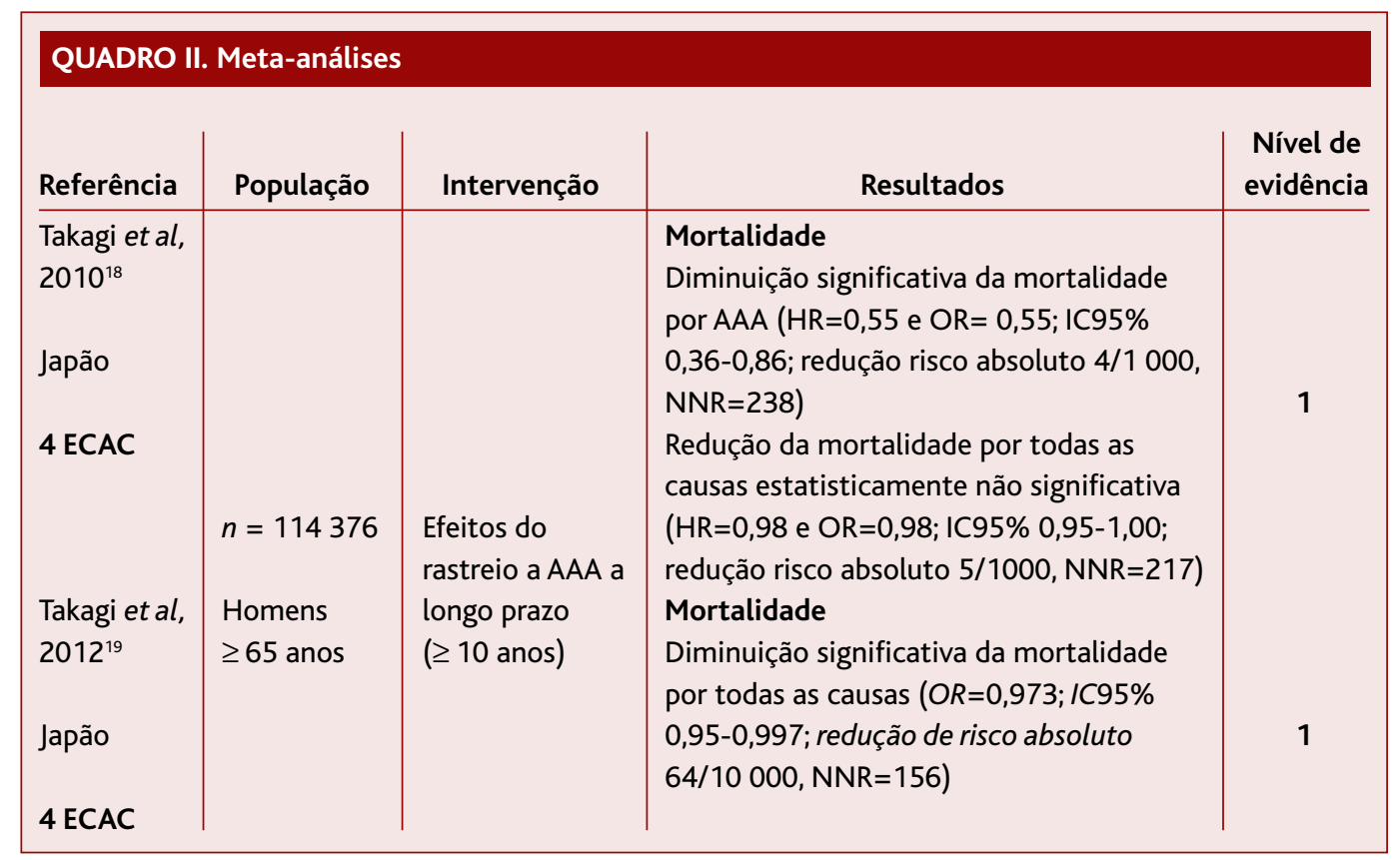

Legenda: AAA - Aneurisma da Aorta Abdominal; ECAC - Ensaio Clínico Aleatorizado e Controlado; HR - Hazard Ratio; IC - Intervalo de Confiança; NNR - Número Necessário para Rastrear; OR - Odds Ratio. 
como também a alguns aspetos da RS da Cochrane, uma vez que nestas revisões foram incluídos dados relativos às mortes de um ECAC (Western Australia) não ajustados para a idade. ${ }^{18,20}$

Foram ainda incluídas 7 NOC, a maioria realizadas no norte da América (quadro III). Ferket et al realizaram uma análise qualitativa de muitas das NOC incluídas nesta revisão, tendo sido aquelas com pontuações de rigor mais elevadas a da USPSTF (79\%), da American College of Cardiology/American Heart Association (ACC/AHA) (63\%) e a do National Screening Committee (NSC) $(41 \%){ }^{21}$ Provavelmente neste sentido, várias organizações adotaram as recomendações da USPSTF, entre as quais a American College of Preventive Medicine (ACPM), a American Academy of Family Physicians (AAFP) e a University of Medicine and Health Sciences (UMHS). ${ }^{6,12-13}$

Apenas duas NOC incluídas foram desenvolvidas com base numa revisão sistemática (ACC e USPSTF), ${ }^{3,7}$ As restantes foram desenvolvidas a partir de uma seleção não sistemática de revisões sistemáticas já realizadas ou de uma pesquisa primária. A ecografia abdominal foi unanimemente recomendada como método de rastreio, sendo que apenas uma NOC (ACC/AHA) ${ }^{3}$ incluiu o exame físico como ferramenta de rastreio em associação à ecografia.

Parece existir um consenso entre as NOC a favor do rastreio ecográfico único nos homens idosos, a maioria recomendando como idade de início do rastreio os 65 anos. Inversamente, não houve unanimidade sobre se uma história de tabagismo deva ou não estar presente, com a NOC da ACC/AHA a recomendar o rastreio em homens que já fumaram (definido como qualquer hábito tabágico ativo ou passivo) e a NOC da USPSTF a exigir nas suas recomendações o consumo de pelo menos 100 cigarros ao longo da vida. ${ }^{3,7}$

Relativamente à história familiar, apesar de a USPSTF não emitir recomendações relativamente a este fator de risco, ele é reconhecido mas considerado de menor impacto comparativamente à idade, o sexo e os hábitos tabágicos. ${ }^{7}$ A USPSTF enfatiza a importância do médico individualizar os cuidados para cada consulente, avaliando os seus fatores de risco, inclusive história familiar, e os potenciais benefícios a obter com o rastreio. ${ }^{7}$

Na abordagem dos AAA de pequenas dimensões não houve concordância entre as NOC ou não eram emiti- das recomendações. À exceção da USPSTF, todas as NOC continham recomendações acerca dos intervalos de vigilância para aneurismas com diâmetros inferiores à indicação para cirurgia eletiva $(5,5 \mathrm{~cm})$. A Society for Vascular Surgery (SVS) e a German Society for Vascular Surgery (GSVS) emitiram recomendações concordantes: em AAA com $<3 \mathrm{~cm}$ não recomendavam mais exames; AAA entre $3-4 \mathrm{~cm}$, ecografia anual; AAA entre $4-4,5 \mathrm{~cm}$, ecografia semestral e AAA $>4,5 \mathrm{~cm}$ sugeriam a referenciação ao especialista vascular. ${ }^{5,22}$

\section{CONCLUSÕES}

Do processo de pesquisa, os autores consideram ter obtido artigos robustos de encontro aos objetivos deste trabalho.

A RS e as MA incluíram ECAC de boa qualidade, com resultados congruentes entre si e orientados para o paciente. Assim, o corpo de evidência encontrado foi robusto em benefício do rastreio do AAA em homens assintomáticos com idades superiores ou iguais a 65 anos, uma vez que demonstrou reduzir a mortalidade por AAA (NE 1) e por todas as causas (NE 2). Esta conclusão vai naturalmente de encontro às NOC elaboradas por entidades americanas e europeias.

No que concerne ao efeito do rastreio do AAA na mortalidade por todas as causas, a evidência mais recente traduz um efeito favorável, mas ainda ficam algumas questões por responder. Todos os ensaios demonstraram reduções pequenas ou não significativas da mortalidade por todas as causas com o rastreio, inclusive o ensaio MASS, considerado o mais importante. Após a publicação deste ECAC, ${ }^{14}$ a equipa de Takagi et al actualizou a sua MA, ${ }^{19}$ concluindo que o rastreio ao AAA diminuía significativamente a longo prazo a mortalidade por todas as causas em homens com idades superiores ou iguais a 65 anos. Uma vez que a mortalidade atribuída ao AAA assoma um pequeno contributo (2\%) para todas as mortes nos homens idosos, será provável que a diminuição da mortalidade por AAA poderá não contribuir diretamente para a redução da mortalidade por todas as causas. Os investigadores Mastracci e Cinà defendem a hipótese de que este resultado poderá ser devido à redução de fatores de risco cardiovasculares associados ao estilo de vida a que os participantes do rastreio estiveram sujeitos. ${ }^{24}$

Os artigos selecionados permitem concluir que o ras- 


\section{QUADRO III. Normas de Orientação Clínica}

\begin{tabular}{|c|c|c|c|}
\hline Referência & País & Recomendações & $\begin{array}{l}\text { Nível de } \\
\text { evidência }\end{array}$ \\
\hline $\begin{array}{l}\text { USPSTF, } \\
2005^{7}\end{array}$ & EUA & $\begin{array}{l}\text { Recomenda o rastreio único através de ecografia abdominal a homens com idades } \\
\text { entre os } 65-75 \text { anos que já tenham fumado* } \\
\text { Sem recomendação a favor ou contra para homens com idades entre os } 65-75 \text { anos } \\
\text { que nunca fumaram } \\
\text { Recomenda contra o rastreio em mulheres }\end{array}$ & $\begin{array}{l}1 \\
3 \\
2\end{array}$ \\
\hline $\begin{array}{l}\text { ACC/AHA } \\
2005^{3}\end{array}$ & EUA & $\begin{array}{l}\text { Recomenda que homens com idades entre os } 65-75 \text { anos que já fumaram** devem ser } \\
\text { submetidos a um exame físico e uma ecografia única de rastreio } \\
\text { Recomenda que homens com idades } \geq 60 \text { anos, que sejam irmãos ou filhos de pacientes } \\
\text { com AAA, devem ser submetidos a um exame físico e ecografia abdominal }\end{array}$ & $\begin{array}{l}1 \\
2\end{array}$ \\
\hline NSC, $2007^{23}$ & $\begin{array}{l}\text { Reino } \\
\text { Unido }\end{array}$ & Recomenda o rastreio único através de ecografia abdominal em homens com 65 anos & 2 \\
\hline $\begin{array}{l}\text { ESVS, } \\
2011^{4}\end{array}$ & Holanda & $\begin{array}{l}\text { Recomenda o rastreio único através de ecografia abdominal aos homens com } 65 \text { anos } \\
\text { Recomenda considerar o rastreio em idades mais jovens para aqueles com alto risco de } \\
\text { AAA } \\
\text { Recomenda considerar o rastreio nos indivíduos com idades > } 50 \text { anos com história } \\
\text { familiar de AAA }\end{array}$ & $\begin{array}{l}1 \\
4 \\
3\end{array}$ \\
\hline $\begin{array}{l}\text { CSVS, } \\
2007^{24}\end{array}$ & Canadá & $\begin{array}{l}\text { Recomenda rastreio a homens com idades entre } 65-75 \text { anos } \\
\text { Recomenda considerar o rastreio através de ecografia em mulheres com > } 65 \text { anos e } \\
\text { múltiplos fatores de risco para AAA (tabagismo, doença cerebrovascular, história } \\
\text { familiar de AAA) } \\
\text { Recomendam contra o rastreio em mulheres com idades > } 65 \text { anos e na população } \\
\text { adulta com idades < } 65 \text { anos }\end{array}$ & $\begin{array}{l}2 \\
5\end{array}$ \\
\hline $\begin{array}{l}\text { GSVS, } \\
2009^{5}\end{array}$ & Alemanha & $\begin{array}{l}\text { Recomenda o rastreio único através de ecografia abdominal a homens com idades } \\
\text { > } 65 \text { anos, especialmente se são ou se já foram fumadores } \\
\text { Recomenda o rastreio único através de ecografia abdominal em indivíduos de todas as } \\
\text { idades com história familiar de AAA } \\
\text { Recomenda o rastreio único através de ecografia abdominal a mulheres com idades } \\
\text { > } 65 \text { anos que sejam ou já tenham sido fumadoras e com antecedentes de doença } \\
\text { cardiovascular }\end{array}$ & $\begin{array}{l}1 \\
3 \\
5\end{array}$ \\
\hline SVS, $2004^{22}$ & EUA & $\begin{array}{l}\text { Recomenda o rastreio único através de ecografia abdominal em todos os homens com } \\
\text { idades } 60-85 \text { anos } \\
\text { Mulheres com idades entre os } 60-85 \text { anos com fatores de risco cardiovasculares } \\
\text { Em todos os indivíduos com idades > } 50 \text { anos e com história familiar de AAA }\end{array}$ & $\begin{array}{l}2 \\
5 \\
5\end{array}$ \\
\hline
\end{tabular}

Legenda: AAA - Aneurisma da Aorta Abdominal; ACC/AHA - American College of Cardiology/American Heart Association; CSVS - Canadian Society for Vascular Surgery; ESVS - European Society for Vascular Society; EUA - Estados Unidos da América; GSVS - German Society for Vascular Surgery; NSC National Screening Committee; SVS - Society for Vascular Surgery; USPSTF - United States Preventive Services Task Force.

* Indivíduos que fumaram pelo menos 100 cigarros ao longo da vida., ${ }^{2,13}$

** Hábitos tabágicos ativos ou passivos.

treio populacional do AAA é marcadamente eficiente, comparando os NNR estimados com os de outros programas de rastreio já estabelecidos. Por exemplo, acer- ca do rastreio do cancro da mama através de mamografia, uma RS da Cochrane em 2011 mostrou uma redução significativa da mortalidade por cancro da 
mama, sem redução significativa da mortalidade por todas as causas após 13 anos. ${ }^{25}$ Assim, em 2.000 mulheres convidadas para o rastreio ao longo de 10 anos, uma teve a sua vida prolongada, 10 foram diagnosticadas com cancro da mama apenas porque realizaram o rastreio, tendo sido tratadas desnecessariamente e mais de 200 experienciaram um stress psicológico importante relacionado com os falsos positivos. ${ }^{25}$ Uma outra RS da Cochrane indicou que o rastreio do cancro colorrectal diminuía em $16 \%$ o risco relativo de mortalidade, num seguimento entre os 8 e 18 anos (1,5/1.000; NNR 671), sem diferenças na mortalidade por todas as causas. ${ }^{26}$ Relativamente ao controverso rastreio do cancro da próstata, o NNR estimado ronda os 1.245 aos 9 anos. ${ }^{27} \mathrm{O}$ rastreio do AAA, num seguimento de 10 a 15 anos, reduziu a mortalidade relacionada com o AAA e a mortalidade por todas as causas com um NNR estimado em 238 (4/1.000) e 156, respetivamente., ${ }^{5,14,18-19,28}$

A ecografia foi usada nos ECAC e nos programas de rastreio realizados, revelando deter as características de um exame de rastreio ideal, sendo simples, seguro, confiável, válido e bem aceite pela população em geral. ${ }^{5,24}$ Apesar de existirem consensos quanto aos intervalos de reavaliação de um AAA em vigilância, baseados nos ensaios clínicos, aguarda-se evidência científica que indique os intervalos ótimos, seguros e custoeficazes. ${ }^{4} \mathrm{Na}$ verdade, a história natural do AAA, a sua formação e expansão não estão completamente compreendidas. A patogénese é complexa e multifatorial, sendo necessários mais estudos.

À luz da evidência atual não parece haver evidência para apoiar o rastreio do AAA em mulheres, uma vez que este não demonstrou diminuir a mortalidade (NE 3). Há que considerar, porém, que apenas um ECAC foi realizado em mulheres (Chichester) e que, atendendo à baixa prevalência de AAA neste sexo, este ensaio pode não ter tido o poder suficiente para detetar qualquer benefício do rastreio. Adicionalmente, o tabagismo, como o principal fator de risco para AAA, tem vindo a aumentar nas mulheres, desconhecendo-se a incidência futura de AAA em mulheres fumadoras. Desta forma, seria pertinente a realização de estudos acerca do benefício do rastreio em mulheres fumadoras.

A história familiar de AAA aumenta o risco de AAA em cerca de duas vezes. ${ }^{1,4} \mathrm{O}$ rastreio de indivíduos com história familiar de AAA poderá ser aconselhável (NE 3), desconhecendo-se a idade a partir da qual o rastreio será mais vantajoso.

Todos os ECAC rastrearam indivíduos aos 65 ou mais anos, como sendo a idade na qual a prevalência de AAA é elevada o suficiente para haver benefício no rastreio, tendo em conta o baixo risco de rotura numa idade anterior e os custos de repetir o rastreio mais tarde. Nenhum estudo avaliou, porém, a idade ótima na qual o benefício é maior em termos de vidas salvas e de custo-benefício.

Um dos aspetos a refletir nos rastreios é a sustentabilidade do seu benefício ao longo dos anos. Isto pode ser ameaçado se, por exemplo, indivíduos com idades $\geq 65$ anos, que tiveram um rastreio negativo, vierem a sofrer uma rotura de um AAA posteriormente. A RS da USPSTF, que incluiu ECAC com um seguimento até aos 5 anos, concluiu que um rastreio ao AAA negativo aos 65 anos virtualmente excluía o risco de uma rotura de AAA, uma vez que a morte por essa complicação seria posteriormente rara, com uma taxa de incidência estimada para um novo AAA de cerca de $0-4 \%$ em 10 anos. ${ }^{2,7}$ Porém, numa análise a longo prazo, dois dos quatro ECAC incluídos nas RS e MA verificaram um aumento das roturas de AAA a partir dos 8 anos de seguimento nos homens que tinham tido um rastreio negativo (especialmente nos indivíduos com diâmetros da aorta no limite superior do valor diagnóstico), com uma taxa de cerca de uma rotura aneurismática em 1.000 por ano. ${ }^{14}$ Esta taxa mantinha-se até aos 13 anos de seguimento e, ainda que sendo baixa em termos absolutos, foi suficiente para diminuir o benefício do rastreio a longo-prazo. Os autores do ECAC MASS concluíram que estes eventos não produziam uma diminuição significativa no benefício do rastreio em reduzir a mortalidade por AAA. ${ }^{14}$ De qualquer forma, a evicção destes eventos poderá passar pela repetição do rastreio ao fim de 10 anos a todos os homens com diâmetros da aorta abdominal normais ou integrar aqueles com diâmetros no limite superior da normalidade em programas da vigilância.

Relativamente aos efeitos laterais do rastreio, o efeito nocivo provavelmente mais importante é o risco de mortalidade associado à intervenção cirúrgica e/ou de complicações. Houve duas vezes mais cirurgias eletivas a AAA no grupo convidado para rastreio do que no controle; no entanto, o número de cirurgias de emergência reduziu para metade..$^{2,-10,14}$ Não se identificaram dife- 
renças nas taxas de mortalidades associadas a estes procedimentos entre o grupo rastreado e o de controlo. ${ }^{14}$

A mortalidade associada à cirurgia de reparação eletiva $(4,2 \%)$, apesar de ser bastante inferior àquela associada a uma rotura de AAA $(34,1 \%)$, não é insignificante. ${ }^{2,9,14}$ Assim, ao reparar um aneurisma, um paciente é exposto a um risco importante quando o seu AAA (mesmo se grande) poderia não ser a causa da sua morte. O balanço entre o risco de rotura e os riscos da cirurgia eletiva (morte e outras complicações) é difícil de julgar em indivíduos assintomáticos. Por outro lado, serão diagnosticados pequenos aneurismas a muitos indivíduos saudáveis aos quais a cirurgia não está recomendada nem existe um tratamento efetivo na redução ou interrupção do crescimento do AAA, o que poderá gerar sentimentos de ansiedade. ${ }^{9}$ Estes indivíduos poderão viver com o peso de um diagnóstico potencialmente fatal e estar sujeitos a uma vigilância periódica, o que poderá afetar negativamente a sua qualidade de vida.

O rastreio pode ainda causar uma sobrecarga ao nível dos serviços de cirurgia vascular, os quais para tornar o rastreio seguro deverão proporcionar tratamentos de reparação cirúrgica com baixas taxas de mortalidade.

Dado que todos os ensaios clínicos decorreram fora de Portugal, a possibilidade de generalizar os resultados para a população portuguesa não é clara.

Apesar da melhor evidência exposta neste trabalho, na recomendação deste rastreio há que considerar várias questões. Os indivíduos alvo terão que ser suficientemente semelhantes àqueles dos ECAC. Por outro lado, o rastreio deve claramente trazer um benefício clinico relevante que ultrapasse os danos e dever-se-á questionar se existirá outro melhor. Há que considerar a aceitabilidade e os valores dos pacientes tendo em conta os possíveis riscos inerentes ao rastreio e ao tratamento. Não interessará a um paciente saber, por exemplo, os benefícios/malefícios do rastreio do AAA se este se negar a ser sujeito a uma intervenção cirúrgica. Estas posições carecem de uma tomada de decisões partilhada entre o doente e o médico. Será ainda necessário aprofundar a análise sobre a qualidade de vida, complicações cirúrgicas, expectativa de vida e relação custo-efetividade, cujos dados existentes parecem ser insuficientes. ${ }^{9}$

\section{AGRADECIMENTOS}

Os autores gostariam de agradecer à Dra. Magda Cardoso e à Dra. Cláudia Melo pela revisão crítica do artigo.

\section{REFERÊNCIAS BIBLIOGRÁFICAS}

1. Desjardins B, Rybicki FJ, Dill KE, Flamm SD, Francois CJ, Gerhard-Herman MD, et al.ACR Appropriateness Criteria ${ }^{\circledR}$ pulsatile abdominal mass, suspected abdominal aortic aneurysm. Reston (VA): American College of Radiology; 2012.

2. Fleming C, Whitlock EP, Beil TL, Lederle FA. Screening for abdominal aortic aneurysm: a best-evidence systematic review for the U.S. Preventive Services Task Force. Ann Intern Med. 2005;142(3):203-11.

3. Hirsch AT, Haskal ZJ, Hertzer NR, Bakal CW, Creager MA, Halperin JL, et al. ACC/AHA 2005 guidelines for the management of patients with peripheral arterial disease (lower extremity, renal, mesenteric, and abdominal aortic): a collaborative report. Bethesda (MD): American College of Cardiology Foundation; 2005.

4. Moll FL, Powell JT, Fraedrich G, Verzini F, Haulon S, Waltham M, et al. Management of abdominal aortic aneurysms clinical practice guidelines of the European Society for Vascular Surgery (ESVS). Eur J Vasc Endovasc Surg. 2011;41 Suppl 1:S1-S58.

5. Eckstein HH, Böckler D, Flessenkämper I, Schmitz-Rixen T, Debus S, Lang W. Ultrasonographic screening for the detection of abdominal aortic aneurysms. Dtsch Arztebl Int. 2009;106(41):657-63.

6. Lim LS, Haq N, Mahmood S, Hoeksema L, ACPM Prevention Practice Committee, American College of Preventive Medicine. Atherosclerotic cardiovascular disease screening in adults: American College of Preventive Medicine position statement on preventive practice. Am J Prev Med. 2011;40(3):381.e1-10.

7. U.S. Preventive Services Task Force. Screening for abdominal aortic aneurysm: recommendation statement. Ann Intern Med. 2005;142(3):198-202.

8. American College of Radiology, American Institute of Ultrasound in Medicine, Society of Radiologists in Ultrasound. ACR-AIUM-SRU practice guideline for the performance of diagnostic and screening ultrasound of the abdominal aorta in adults. Reston (VA): American College of Radiology; 2010.

9. Cosford PA, Leng GC. Screening for abdominal aortic aneurysm. Cochrane Database Syst Rev. 2007;(2):CD002945.

10. Lindholt JS, Norman P. Screening for abdominal aortic aneurysm reduces overall mortality in men: a meta-analysis of the mid- and longterm effects of screening for abdominal aortic aneurysms. Eur J Vasc Endovasc Surg. 2008;36(2):167-71.

11. Canadian Association Radiologists. Diagnostic imaging referral guidelines, section E: cardiovascular. Vancouver: CAR; 2013. Available from: http://www.car.ca/uploads/standards\%20guidelines/car-referralguidelines-e-en-20121011.pdf

12. American Academy of Family Physicians. Summary of recommendations for clinical preventive services. Leawood (KS): AAFP; 2012.

13. University of Michigan Health System. Adult clinical preventive care. Ann Arbor (MI): UMHS; 2011

14. Thompson SG, Ashton HA, Gao L, Buxton MJ, Scott RA, Multicentre Aneurysm Screening Study (MASS) Group. Final follow-up of the Multicentre Aneurysm Screening Study (MASS) randomized trial of abdo- 
minal aortic aneurysm screening. Br J Surg. 2012;99(12):1649-56.

15. SBU, Swedenborg J. Screening for abdominal aortic aneurysm. Stockholm: Swedish Council on Health Technology Assessment; 2008. Available from: www.sbu.se/upload/Publikationer/Content0/3/Screening_Abdominal_Aortic_Aneurysm_200804.pdf

16. Sociedade Portuguesa de Angiologia e Cirurgia Vascular. Aorta é vida [website]. Lisboa: Círculo Médico; 2009. Available from: www.aortaevida.com

17. OCEBM Levels of Evidence Working Group. The Oxford 2011 levels of evidence. Oxford Centre for Evidence-Based Medicine; 2011. Available from: $w$ ww.cebm.net/index.aspx?o=5653

18. Takagi $\mathrm{H}$, Goto $\mathrm{SN}$, Matsui M, Manabe H, Umemoto T. A further metaanalysis of population- based screening for abdominal aortic aneurysm. J Vasc Surg. 2010;52(4):1103-8.

19. Takagi H, Niwa M, Mizuno Y, Goto SN, Umemoto T. The Last Judgment upon abdominal aortic aneurysm screening. Int J Cardiol. 2013;167(5):2331-2.

20. Dabare D, Lo TT, McCormack DJ, Kung VW. What is the role of screening in the management of abdominal aortic aneurysms? Interact Cardiovasc Thorac Surg. 2012;14(4):399-405.

21. Ferket BS, Grootenboer N, Colkesen EB, Visser JJ, van Sambeek MR, Spronk S, et al. Systematic review of guidelines on abdominal aortic aneurysm screening. J Vasc Surg. 2012;55(5):1296-304.

22. Kent KC, Zwolak RM, Jaff MR, Hollenbeck ST, Thompson RW, Schermerhorn $M L$, et al. Screening for abdominal aortic aneurysm: a consensus statement. J Vasc Surg. 2004;39(1):267-9.

23. National Screening Committee. Abdominal aortic aneurysm screening.
NSC; 2007. Available from: http://aaa.screening.nhs.uk/screening

24. Mastracci TM, Cinà CS, Canadian Society for Vascular Surgery. Screening for abdominal aortic aneurysm in Canada: review and position statement of the Canadian Society for Vascular Surgery. J Vasc Surg. 2007;45(6):1268-76.

25. Gøtzsche PC, Nielsen M. Screening for breast cancer with mammography. Cochrane Database Syst Rev. 2011;(1):CD001877.

26. Hewitson P, Glasziou P, Irwig L, Towler B, Watson E. Screening for colorectal cancer using the faecal occult blood test, Hemoccult. Cochrane Database Syst Rev. 2007;(1):CD001216.

27. Loeb S, Vonesh EF, Metter EJ, Carter HB, Gann PH, Catalona WJ. What is the true number needed to screen and treat to save a life with prostate-specific antigen testing? J Clin Oncol. 2011;29(4):464-7.

28. Cornuz J, Sidoti Pinto C, Tevaerai H, Egger M. Risk factors for asymptomatic abdominal aortic aneurysm. Eur J Public Health. 2004;14(4):3439.

\section{CONFLITOS DE INTERESSES}

Os autores declaram não possuir conflitos de interesse.

\section{ENDEREÇO PARA CORRESPONDÊNCIA}

Vítor Bruno Portela Lopes Cardoso

Rua João Gomes Leite n. ${ }^{\circ}$ 37, Real. 4700-276 Braga

E-mail:muxmail@gmail.com

Recebido em 20/09/2013

Aceite para publicação em 20/08/2014

\section{ABSTRACT}

\section{SCREENING FOR ABDOMINAL AORTIC ANEURYSM - AN EVIDENCE-BASED REVIEW}

Objective: Review the evidence of mortality and adverse effects of screening for abdominal aortic aneurysm (AAA) in asymptomatic adults.

Data Sources: National Guideline Clearinghouse, Guidelines Finder, Canadian Medical Association Practice Guidelines, Cochrane, DARE, Bandolier, MEDLINE and Index of Portuguese Medical Journals.

Methods: We conducted a search for meta-analyses (MA), systematic reviews (SR), randomized clinical trials (RCT) and clinical practice guidelines (CPG), published between 01/01/2003 and 03/23/2013 in the Portuguese, Spanish, English and French languages, using the MeSH terms abdominal aortic aneurysm and mass screening. We used the Oxford Centre for EvidenceBased Medicine (CEBM) scale for assessment of levels of evidence (LE).

Results: Of 337 articles obtained, 10 met the inclusion criteria (2 MA, 1 SR and 7 CPG). The SR and the MA selected showed that screening for AAA significantly decreased mortality in men aged between 65-75 years old, but not in women. A reduction in all-cause mortality was observed in one MA, which estimated a number needed to screen of 156 . This is lower than the number needed to screen in current cancer screening programs. Screening doubled the number of elective surgeries and decreased the number of emergency surgeries for ruptured AAA by half.

Conclusions: Consistent, good quality evidence demonstrated a reduction in mortality from AAA by screening asymptomatic men aged $\geq 65$ years (LE1). The treatment of AAA is associated with significant risk of death and surgical complications, which may be acceptable in individuals at high risk.

Keywords: Abdominal Aortic Aneurysm; Mass Screening; Mortality, Side Effects. 